\section{Intensity Estimation from Shot-Noise Data}

Raúl E. Sequeira and John A. Gubner

Abstract-The estimation of the intensity function of a Poisson-driven shot-noise process is addressed using a regularization technique, where the data is modeled as a signal term plus a signal-dependent noise term. A new data-based method for selecting a pair of regularization parameters is presented and compared with the minimum unbiased risk method. The detail in the intensity function can be recovered by both methods, but the new method does a better job at suppressing spurious oscillations.

\section{INTRODUCTION}

We consider the estimation of the intensity function of an inhomogeneous Poisson process from a realization of the point process that has been filtered by a linear time-invariant system with impulse response $h(t)$. More precisely, let $\left\{T_{\nu}\right\}$ denote the occurrence times of a Poisson process with intensity $\lambda(\tau)$. The random system input can be viewed as $\sum_{\nu} \delta\left(\tau-T_{\nu}\right)$; the system output is the shot-noise process $z(t):=\sum_{\nu} h\left(t-T_{\nu}\right)$. The purpose of this paper is to present a new method to estimate $\lambda(\tau)$ for $\tau \in \mathcal{T}_{1}$ based on observing $z(t)$ for $t \in \mathcal{T}_{2} ; \mathcal{T}_{1}$ and $\mathcal{T}_{2}$ are finite closed intervals.

To outline our approach to this problem, let $\mu(t):=\mathrm{E}[z(t)]$ and $\sigma^{2}(t):=E\left[|z(t)-\mu(t)|^{2}\right]$; it is well known [10, eq. (16-84)] that $\mu(t)=\int h(t-\tau) \lambda(\tau) d \tau$ and $\sigma^{2}(t)=\int|h(t-\tau)|^{2} \lambda(\tau) d \tau$. Setting $v(t):=z(t)-\mu(t)$, we clearly have

$$
z(t)=\mu(t)+v(t)=\int h(t-\tau) \lambda(\tau) d \tau+v(t),
$$

where $v(t)$ is a "noise" term with zero mean and variance $\sigma^{2}(t)$. The importance of (1.1) is that it suggests a new way of looking at our estimation problem: estimating $\lambda$ is now seen to be equivalent to finding an approximate solution to a Fredholm integral equation of the first kind when the data are noisy. Although many approaches have been proposed for solving this type of problem, e.g., [3], [8], [9], [12], [13], it is clear that the "noise" statistics in (1.1) depend on the unknown $\lambda$; this is quite different from the models usually considered in the literature, in which the noise statistics do not depend on the function to be estimated.

The problem of estimating $\lambda$ when the data is given by $y_{\nu}=$ $T_{\nu}+\Sigma_{\nu}$, where the $\varepsilon_{\nu}$ 's are i.i.d. random variables, independent of the $T_{\nu}$ 's, and with known density, has been addressed widely using the expectation-maximization algorithm [4], [6]; a related density-deconvolution problem has been addressed using kerneldensity estimates [5], [11]. To the best of our knowledge, the problem of estimating $\lambda$ from shot-noise data has not been addressed before.

\section{Preliminary CONSIDERations}

Let $\mathcal{H}_{i}:=L^{2}\left(\mathcal{T}_{i}\right), i=1,2$. For $r \in \mathcal{H}_{1}$, we define $H: \mathcal{H}_{1} \rightarrow \mathcal{H}_{2}$ by $(H r)(t):=\int_{\tau} h(t-\tau) r(\tau) d \tau$. Note that either of the following two setups permits us to write $\mu(t)=(H \lambda)(t)$ for $t \in \mathcal{T}_{2}$. In the first setup, $\lambda(\tau)$ is nonzero only for $\tau$ in some closed interval $\mathcal{T}_{1}$, and $\mathcal{T}_{2}$ is a finite closed interval containing $\mathcal{T}_{1}$. In the second setup, $\lambda$ can be an infinite-duration signal, but both $\lambda$ and $h$ must be causal; in this case, we take $\mathcal{T}_{1}=\mathcal{T}_{2}=[0, T]$ for some finite $T$.

Manuscript received September 3, 1993; revised November 10, 1994. This work was supported by the Air Force Office of Scientific Research under Grant F49620-92-J-0305. This work was presented in part at the SPIE Conference on Image Reconstruction and Restoration, San Diego, CA, July 1994.

The authors are with the Department of Electrical and Computer Engineering, University of Wisconsin, Madison, WI 53706 USA

IEEE Log Number 9411202.
We can now rewrite (1.1) as $z=H \lambda+v$, and we consider obtaining an approximate solution via Tikhonov's method of regularization [3]; from a variational approach, this method leads to the consideration of the smoothing functional $f^{\alpha}(r, z):=\| H r-$ $z \|_{\mathcal{H}_{2}}^{2}+\alpha J(r)$, where $r \in \mathcal{H}_{1}$; the term $J(r)$ is called a stabilizing functional. For fixed $\alpha$ and given $z$, the regularized solution is $\arg \min _{r \in \mathcal{H}_{1}} f^{\alpha}(r, z)$, and is found to be the $r$ satisfying

$$
2 H^{*} H r+\left.\alpha \nabla J\right|_{r}=2 H^{*} z,
$$

where $H^{*}$ denotes the adjoint operator of $H$, and $\left.\nabla J\right|_{r}$, the gradient of $J$ at $r$.

For computational tractability, we consider the minimization of $f^{\alpha}(\cdot, z)$ over a finite-dimensional subspace $\mathcal{B} \subset \mathcal{H}_{1}$. This is known as the regularized Ritz approach [3]. We also assume that any $r \in \mathcal{B}$ is differentiable, and we let $\dot{r}$ denote its derivative. We take $J(r):=\|r\|_{\mathcal{H}_{1}}^{2}+c\|\dot{r}\|_{\mathcal{H}_{1}}^{2}$, for some $c>0$ to be selected later. The reason for including the norm of the derivative in the stabilizing functional is that it helps prevent spurious oscillations in the solutions.

\section{A. Sampled Data}

We now assume that we have only $z\left(t_{k}\right)=\mu\left(t_{k}\right)+v\left(t_{k}\right)$, for some design points $\left\{t_{k}\right\}_{k=1}^{K} \subset \mathcal{T}_{2}$. Then, since $\|H r-z\|_{\mathcal{H}_{2}}^{2}$ cannot be computed, a new smoothing functional is introduced below.

Notation: For $u \in \mathcal{H}_{2}$, we write $u:=\left[u\left(t_{1}\right), \cdots, u\left(t_{K}\right)\right]^{\prime}$; this allows us to rewrite the sampled data as $\boldsymbol{z}=\boldsymbol{\mu}+\boldsymbol{v}$. We define an inner product on $\mathbb{R}^{K}$ by $\langle\boldsymbol{q}, \boldsymbol{p}\rangle_{W}:=\boldsymbol{p}^{\prime} W \boldsymbol{q}$, where $W$ is a $K \times \boldsymbol{K}$, diagonal matrix of quadrature weights; we then approximate $\langle p, q\rangle_{\mathcal{H}_{2}}$ by $\langle\boldsymbol{p}, \boldsymbol{q}\rangle_{w}$

\section{B. Finite-Dimensional Approximation}

Let $\mathcal{B}:=\operatorname{span}\left\{B_{m}\right\}_{m=1}^{M}$, where $\left\{B_{m}\right\}_{m=1}^{M}$ is a set of linearlyindependent, differentiable elements in $\mathcal{H}_{1}$. We define the $M \times M$ matrices $E$ and $F$ with elements $E_{m, n}:=\left\langle B_{m}, B_{n}\right\rangle_{\mathcal{H}_{1}}$ and $F_{m, n}:=\left\langle\dot{B}_{m}, \dot{B}_{n}\right\rangle_{\mathcal{H}_{1}}$; note that $E$ is nonsingular since the $\left\{B_{m}\right\}_{m=1}^{M}$ are linearly independent.

Notation: For $r \in \mathcal{B}$, we let $\underline{r} \in \mathbb{R}^{M}$ denote the "coordinate" vector such that $r=\sum_{m=1}^{M} r_{m} B_{m}$. Then, for $a, b \in \mathcal{B},\langle a, b\rangle_{\mathcal{H}_{1}}=$ $\underline{b}^{\prime} E \underline{a}$ and $\langle\dot{a}, \dot{b}\rangle \mathcal{H}_{1}=\underline{b}^{\prime} F \underline{a}$. We let the pair $\left(\mathbb{R}^{M},\langle\cdot, \cdot\rangle_{E}\right)$ denote $\mathbb{R}^{M}$ equipped with the inner product $\langle\underline{a}, \underline{b}\rangle_{E}:=\underline{b^{\prime}} E \underline{a}$, and we let $\|\cdot\|_{E}$ denote the corresponding norm.

Let $G$ be the $K \times M$ design matrix with elements $G_{k, m}:=$ $\left(H B_{m}\right)\left(t_{k}\right)$; then, for $r \in \mathcal{B},\left[(H r)\left(t_{1}\right), \cdots,(H r)\left(t_{K}\right)\right]^{\prime}=G r$. We define the mapping $\tilde{H}$ from $\left(\mathbb{R}^{M},\langle\cdot, \cdot\rangle_{E}\right)$ into $\left(\mathbb{R}^{K},\langle\cdot, \cdot\rangle_{W}\right)$ by $\tilde{H} \underline{r}:=G \underline{r}$, and observe that for $r \in \mathcal{B}, J(r)=\underline{r}^{\prime} D \underline{r}=: \tilde{J}(\underline{r})$, where $D:=E+c F$. We now replace $f^{\alpha}(r, z)$ by $\tilde{f}^{\alpha}(\underline{r}, \boldsymbol{z}):=\| \tilde{H} \underline{r}-$ $\boldsymbol{z} \|_{W}^{2}+\alpha \tilde{J}(\underline{r})$. As our estimate of $\lambda$ we take $\tilde{\lambda}=\sum_{m=1}^{M} \tilde{\lambda}_{m} \bar{B}_{m}$, where $\underline{\tilde{\lambda}}$ denotes the minimizer of $\tilde{f}^{\alpha}(\cdot, \boldsymbol{z})$ over $\mathbb{R}^{M}$. Clearly, $\underline{\tilde{\lambda}}$ is the unique solution of [cf. (2.1)]

$$
2 \tilde{H}^{*} \tilde{H} \underline{r}+\left.\alpha \nabla \tilde{J}\right|_{\underline{r}}=2 \tilde{H}^{*} z .
$$

Theorem 2.1: The unique solution of (2.2), or equivalently, the unique minimizer of $\tilde{f}^{\alpha}(\cdot, z)$, is

$$
\underline{\tilde{\lambda}}:=(L+\alpha D)^{-1} G^{\prime} W \boldsymbol{z}
$$

where $L:=G^{\prime} W G$. Note that $\underline{\tilde{\lambda}}$ is linear in the data $z$.

Proof: $\tilde{H}^{*}$ has the matrix representation $E^{-1} G^{\prime} W$, and $\left.\nabla \tilde{J}\right|_{\underline{r}}=2 E^{-1} D_{\underline{r}}$; using these to rewrite (2.2), and then multiplying by $E$, we obtain $L \underline{r}+\alpha D \underline{r}=G^{\prime} W \boldsymbol{z}$, from which (2.3) follows. $\square$ 


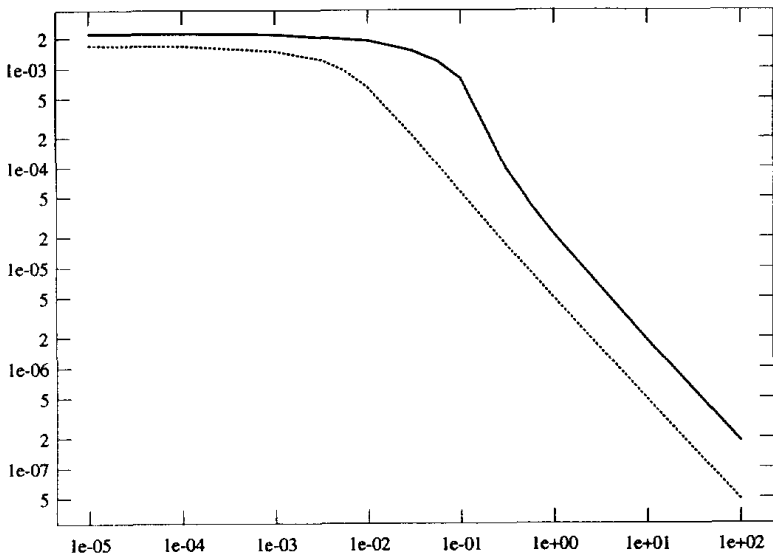

Fig. 1. Example 1: $P_{1}(c)$ (solid line) and $P_{3}(c)$ (dotted line).

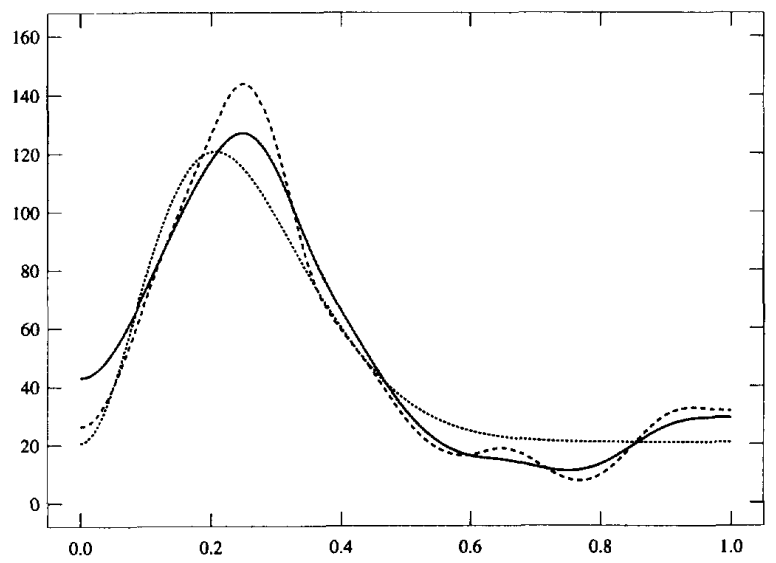

Fig. 2. Example 1: $\tilde{\lambda}$ corresponding to $\left(c_{1}, \alpha_{1}\right)$ (solid line), $\tilde{\lambda}$ corresponding to $\left(c_{3}, \alpha_{3}\right)$ (dashed line), and $\lambda$ (dotted line).

\section{A New CRITERION for ChOOSING $\alpha$}

Recall that $\mu=H \lambda$ and that the data $z=\mu+v$. We now let $\tilde{\mu}:=H \tilde{\lambda}$, and since $\tilde{\lambda} \in \mathcal{B}$, we can write $\tilde{\boldsymbol{\mu}}=\tilde{H} \underline{\tilde{\lambda}}=G \underline{\tilde{\lambda}}$. The difference $\boldsymbol{\mu}-\tilde{\mu}$ is called the prediction error; we now introduce the filtered prediction error $\tilde{H}^{*}(\boldsymbol{\mu}-\tilde{\boldsymbol{\mu}})=\tilde{H}^{*} \boldsymbol{\mu}-\tilde{H}^{*} \tilde{\boldsymbol{\mu}}$, and denote by $R_{1}(\alpha)$ the square of its norm, $R_{1}(\alpha):=\left\|\tilde{H}^{*} \mu-\tilde{H}^{*} \tilde{\mu}\right\|_{E}^{2}$. The motivation for considering $R_{1}(\alpha)$ originates from (2.2): the leastsquares solutions to $\tilde{H} \underline{r}=z$ are the solutions to $\tilde{H}^{*} \tilde{H} \underline{r}=\tilde{H}^{*} z$ (i.e., the solutions to (2.2) when $\alpha=0$ ). Hence, $R_{1}(\alpha)$ is a measure of the deviation of the corresponding $\underline{\hat{\lambda}}$ from being a least-squares solution for the noiseless case when $z=\boldsymbol{\mu}$. A natural way for selecting $\alpha$ would be to choose it as the minimizer of the mean $=$ squared error $\mathrm{E}\left[R_{1}(\alpha)\right]$. Since $\mathrm{E}\left[R_{1}(\alpha)\right]$ cannot be computed in practice, we use $R_{2}(\alpha):=\left\|\tilde{H}^{*} z-\tilde{H}^{*} \tilde{\mu}\right\|_{E}^{2}$ to construct an unbiased estimate of $E\left[R_{1}(\alpha)\right]$, denoted by $\hat{R}_{1}(\alpha)$; in Section $\mathrm{V}$ we obtain an estimate of $\hat{R}_{1}(\alpha)$, which we use in Section VI to choose $\alpha$ and $c$ (recall that $D=E+c F)$.

Recalling (2.3) and that $\tilde{\mu}=G \underline{\underline{\lambda}}$, and using the matrix representation of $\hat{H}^{*}, E^{-1} G^{\prime} W$, we can write $R_{1}(\alpha)=\left(A_{2} \mu-\right.$ $\left.A_{1} z\right)^{\prime} E^{-1}\left(A_{2} \mu-A_{1} z\right)$, where $A_{1}:=L(L+\alpha D)^{-1} G^{\prime} W$ and $A_{2}:=G^{\prime} W$. Similarly, $R_{2}(\alpha)=z^{\prime}\left(A_{2}-A_{1}\right)^{\prime} E^{-1}\left(A_{2}-A_{1}\right) z$.

Theorem 3.1: An unbiased estimate of $\mathrm{E}\left[R_{1}(\alpha)\right]$ is given by

$$
\hat{R}_{1}(\alpha):=R_{2}(\alpha)-\operatorname{tr}\left[E^{-1} A_{2} \Gamma A_{2}^{\prime}\right]+2 \operatorname{tr}\left[E^{-1} A_{2} \Gamma A_{1}^{\prime}\right] \text {, }
$$

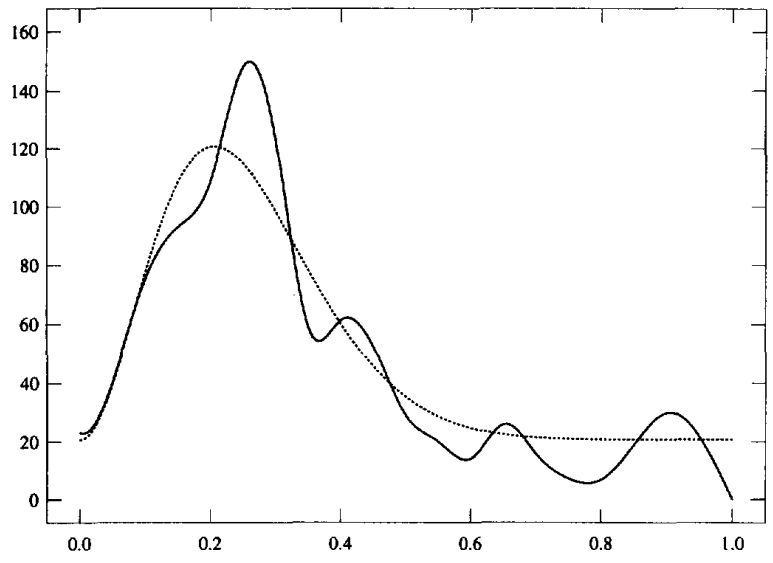

Fig. 3. Example 1: $\tilde{\lambda}$ corresponding to $(c, \alpha)=\left(0, P_{1}(0)\right)$ (solid line) and $\lambda$ (dotted line).

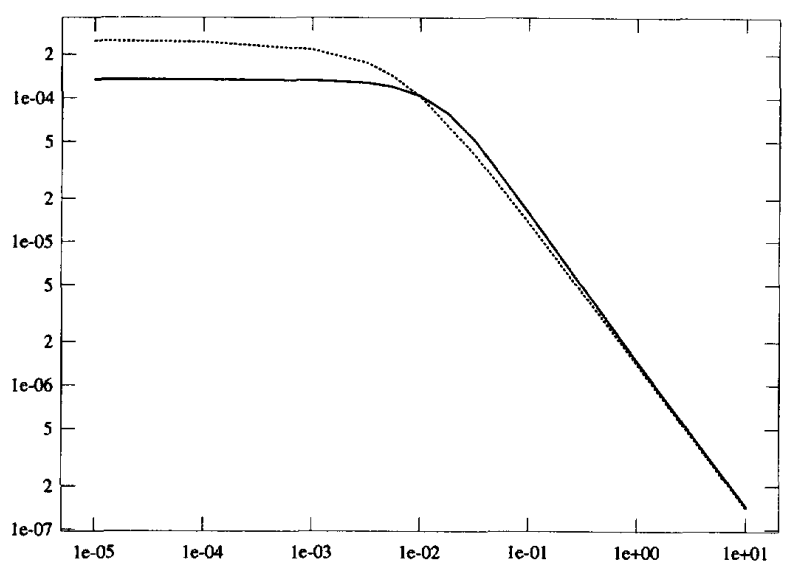

Fig. 4. Example 2: $P_{1}(c)$ (solid line) and $P_{3}(c)$ (dotted line).

where $\Gamma:=E\left[v v^{\prime}\right]$ denotes the covariance matrix of the noise samples.

Proof: Recalling that $z=\mu+v$

$$
R_{1}(\alpha)=\left[\left(A_{2}-A_{1}\right) \mu-A_{1} v\right]^{\prime} E^{-1}\left[\left(A_{2}-A_{1}\right) \mu-A_{1} v\right] .
$$

Then, since the noise samples have zero mean, an easy calculation shows that

$$
\begin{aligned}
\mathrm{E}\left[R_{1}(\alpha)\right]= & \boldsymbol{\mu}^{\prime}\left(A_{2}-A_{1}\right)^{\prime} E^{-1}\left(A_{2}-A_{1}\right) \\
& \boldsymbol{\mu}+\operatorname{tr}\left[E^{-1} A_{1} \Gamma A_{1}^{\prime}\right] .
\end{aligned}
$$

Another easy calculation shows that

$$
\begin{aligned}
\mathrm{E}\left[R_{2}(\alpha)\right]= & \boldsymbol{\mu}^{\prime}\left(A_{2}-A_{1}\right)^{\prime} E^{-1}\left(A_{2}-A_{1}\right) \boldsymbol{\mu} \\
& +\operatorname{tr}\left[E^{-1}\left(A_{2}-A_{1}\right) \Gamma\left(A_{2}-A_{1}\right)^{\prime}\right] .
\end{aligned}
$$

Since $\operatorname{tr}\left[E^{-1} A_{2} \Gamma A_{1}^{\prime}\right]=\operatorname{tr}\left[A_{2} \Gamma A_{1}^{\prime} E^{-1}\right]=\operatorname{tr}\left[E^{-1} A_{1} \Gamma A_{2}^{\prime}\right]$, one can expand the second term on the right-hand side of (3.3) and verify, using (3.1) and (3.2), that $\mathrm{E}\left[\hat{R}_{1}(\alpha)\right]=\mathrm{E}\left[R_{1}(\alpha)\right]$.

\section{Existing Criteria for Choosing $\alpha$}

The criteria in this section are developed by considering the prediction error $\boldsymbol{\mu}-\tilde{\mu}$ 


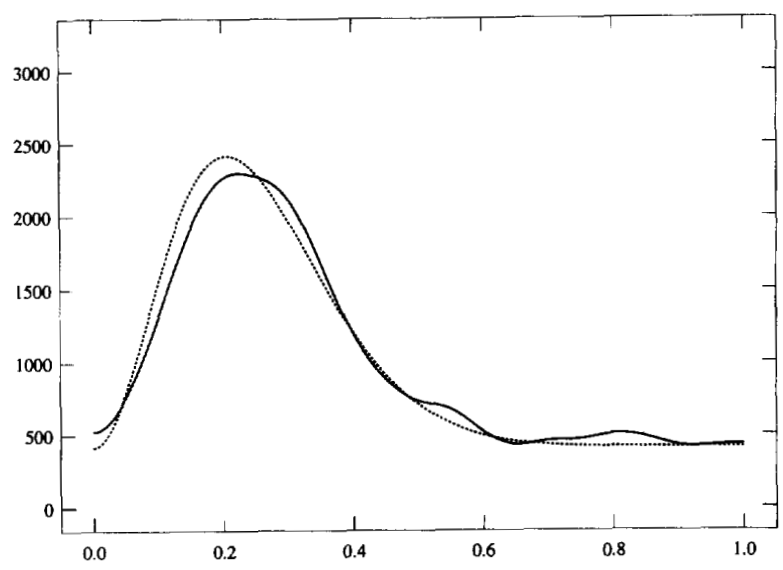

Fig. 5. Example 2: $\tilde{\lambda}$ corresponding to $\left(c_{1}, \alpha_{1}\right)$ (solid line) and $\lambda$ (dotted line).

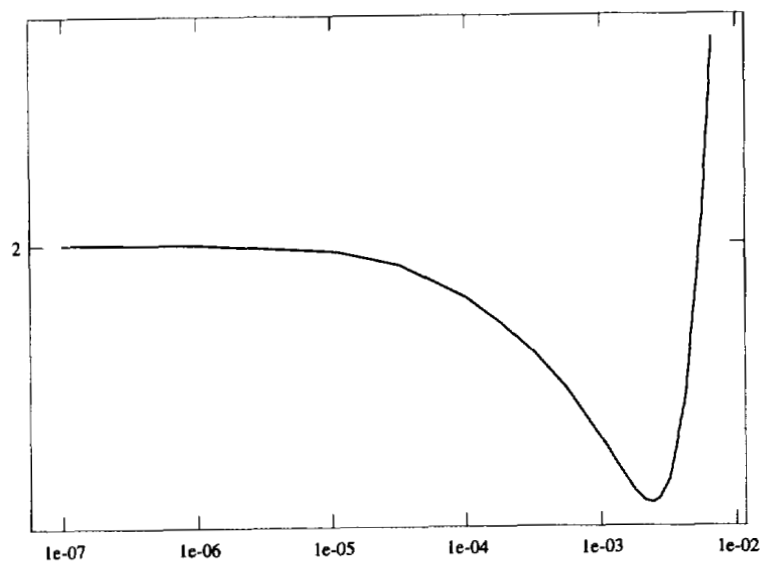

Fig. 6. Example 3: $P_{1}(c)$.

\section{A. The Unbiased Risk Method}

In a very similar way, let $R_{3}(\alpha):=\|\boldsymbol{\mu}-\tilde{\boldsymbol{\mu}}\|_{W}^{2}$ and

$$
\hat{R}_{3}(\alpha):=R_{4}(\alpha)-\operatorname{tr}[W \Gamma]+2 \operatorname{tr}[W A \Gamma],
$$

where $R_{4}(\alpha):=\|z-\tilde{\mu}\|_{W}^{2}=\|(I-A) z\|_{W}^{2}$ and $A:=G(L+$ $\alpha D)^{-1} G^{\prime} W$. Note that $\tilde{\boldsymbol{\mu}}=A \boldsymbol{z}=G \underline{\tilde{\lambda}}$. It is easy to verify that $\mathrm{E}\left[\hat{R}_{3}(\alpha)\right]=E\left[R_{3}(\alpha)\right]$; i.e., $\hat{R}_{3}(\alpha)$ is an unbiased estimate of the mean-squared error $\left[R_{3}(\alpha)\right]$. The method of unbiased risk [9] consists of choosing $\alpha$ as the minimizer of $\hat{R}_{3}(\cdot)$. In Section $\mathrm{V}$ we obtain an estimate of $\hat{R}_{3}$, which we use in Section VI to choose $\alpha$ and $c$.

\section{B. The Generalized Cross-Validation Method}

The method of choosing $\alpha$ as the minimizer of $R_{5}(\alpha):=$ $R_{4}(\alpha) /(\operatorname{tr}[W(I-A)])^{2}$ is known as generalized cross-validation. It is designed [2] under the assumption that the noise samples are uncorrelated, which is not the case in (1.1). We have found that this choice of $\alpha$ is quite unsatisfactory in our application, and we do not give it further consideration. See [13, p. 65].

\section{The Estimate of $\Gamma$}

Both $\hat{R}_{1}(\alpha)$ and $\hat{R}_{3}(\alpha)$ [cf. (3.1) and (4.1)] depend on $\Gamma$, the covariance matrix of the noise samples, where $\Gamma_{i, j}=\int h\left(t_{i}-\right.$ $\tau) h\left(t_{j}-\tau\right) \lambda(\tau) d \tau[10$, eq. (16-86)]. Since $\Gamma$ depends on the

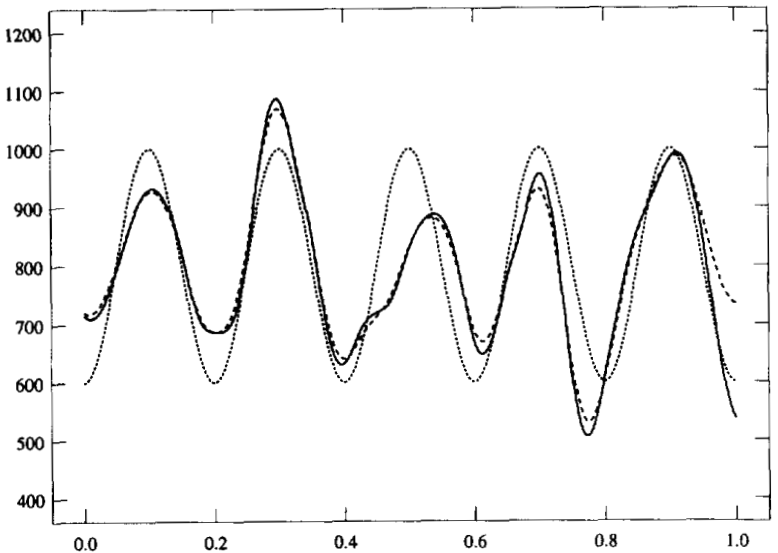

Fig. 7. Example 3: $\tilde{\lambda}$ corresponding to $\left(c_{1}, \alpha_{1}\right)$ (solid line), $\tilde{\lambda}$ corresponding to $\left(c_{3}, \alpha_{3}\right)$ (dashed line), and $\lambda$ (dotted line).

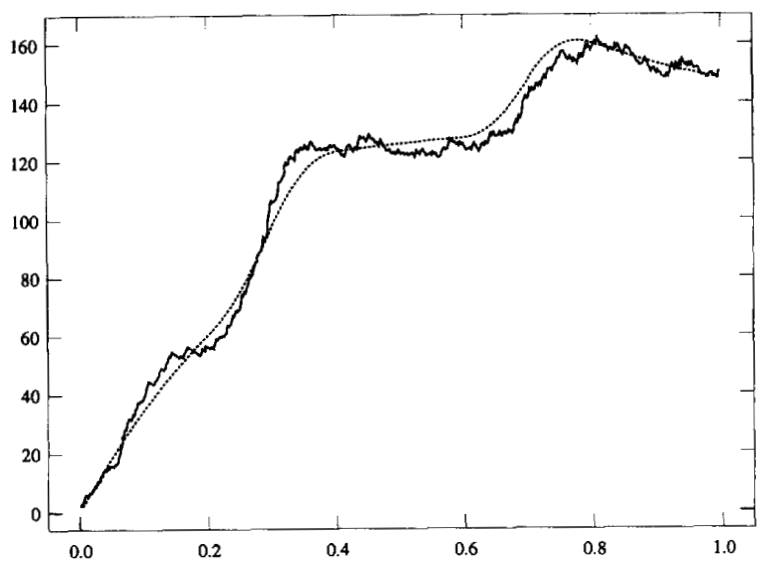

Fig. 8. Example 4: $z(t)$ (solid line) and $\mu(t)$ (dotted line).

unknown $\lambda$, we let $\tilde{\Gamma}$ denote the estimate of $\Gamma$ obtained using the following data-based method. For given values of $c$ and $\alpha$, the estimate of $\lambda$ is $\tilde{\lambda}$, where $\tilde{\lambda}$ is computed using (2.3); we then obtain $\tilde{\Gamma}$ by substituting $\tilde{\lambda}$ in place of $\lambda$ in the expression for $\Gamma_{i, j}$. Since $\hat{\lambda}$ depends on $\alpha$ and $c$, so does $\tilde{\Gamma}$.

We now estimate $\hat{R}_{1}(\alpha)$ by $\hat{R}_{1}(\alpha):=R_{2}(\alpha)-\operatorname{tr}\left[E^{-1} A_{2} \tilde{\Gamma} A_{2}^{\prime}\right]+$ $2 \operatorname{tr}\left[E^{-1} A_{2} \tilde{\Gamma} A_{1}^{\prime}\right]$, and we estimate $\hat{R}_{3}(\alpha)$ by $\tilde{R}_{3}(\alpha):=R_{4}(\alpha)-$ $\operatorname{tr}[W \tilde{\Gamma}]+2 \operatorname{tr}[W A \tilde{\Gamma}]$.

\section{A GRaphical Data-Based Method for ChOOSING $\alpha$ AND $c$}

For the purpose of estimating $\lambda$ via (2.3), we need to choose a value for $\alpha$ and $c$. To this end, we make the following observations. When $c=0$, we have found that plots of $\tilde{R}_{i}(\alpha), i=1,3$, show a unique global minimum and no local minima. If we slightly increase $c$, the position of this minimizer moves continuously. As $c$ increases, it sometimes happens that an additional local minimum appears in plots of $\tilde{R}_{i}(\alpha)$, and it may eventually become the global minimum as $c$ continues to increase. Since we have found that such late-appearing global minimizers are not useful for us, we define $P_{i}(c)$ to be the continuous path of minimizer positions traced out by the original minimizer as $c$ increases from zero. Typical $P_{i}(c)$-curves look like those shown in Figs. 1 and 4; for small values of $c$, the curves are "flat," and $\left.P_{i}(c) \approx \arg \min \tilde{R}_{i}(\cdot)\right|_{c=0}$; for large values of $c$, $c P_{i}(c) \approx p$ for some constant $p$. This behavior of $P_{i}(c)$ for small 
TABLE I

PARAMETERS FOR EXAMPLES $1-3$

\begin{tabular}{ccccccccc}
\hline Example & $\lambda$ & $\Lambda$ & $P_{i}$ & $c_{1}$ & $\alpha_{1}$ & $c_{3}$ & $\alpha_{3}$ & $\tilde{\lambda}$ \\
\hline 1 & Fig. 2 & 50 & Fig. 1 & 1 & $2.080 \times 10^{-5}$ & 0.032 & $2.119 \times 10^{-4}$ & Fig. 2 \\
2 & Fig. 5 & 1000 & Fig. 4 & 0.1 & $1.611 \times 10^{-5}$ & 0.032 & $4.039 \times 10^{-5}$ & Fig. 5 \\
3 & Fig. 7 & 800 & Fig. 6 & 0.001 & $1.976 \times 10^{-4}$ & 0.0056 & $7.167 \times 10^{-5}$ & Fig. 7 \\
\hline
\end{tabular}

TABLE II

PARAMETERS For EXAMPLES 4-6

\begin{tabular}{cccccccc}
\hline Example & $h(t)$ & $z$ and $\mu$ & $c_{1}$ & $\alpha_{1}$ & $c_{3}$ & $\alpha_{3}$ & $\tilde{\lambda}$ \\
\hline 4 & $\exp (-3 t)$ & Fig. 8 & 0.56 & $3.162 \times 10^{-6}$ & 0.01 & $3.659 \times 10^{-5}$ & Fig. 9 \\
5 & $\exp (-3 t) \cos (5 \pi t)$ & Fig. 10 & 1 & $5.757 \times 10^{-6}$ & 1 & $6.012 \times 10^{-7}$ & Fig. 11 \\
6 & $\exp (-3 t) \sin (5 \pi t)$ & Fig. 12 & 0.1 & $5.890 \times 10^{-5}$ & 0.1 & $4.104 \times 10^{-5}$ & Fig. 13 \\
\hline
\end{tabular}

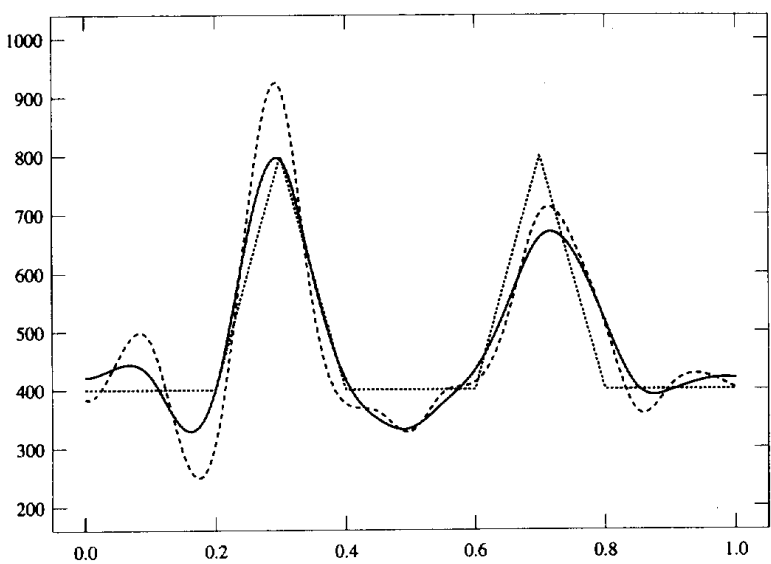

Fig. 9. Example 4: $\bar{\lambda}$ corresponding to $\left(c_{1}, \alpha_{1}\right)$ (solid line), $\tilde{\lambda}$ corresponding to $\left(c_{3}, \alpha_{3}\right)$ (dashed line), and $\lambda$ (dotted line).

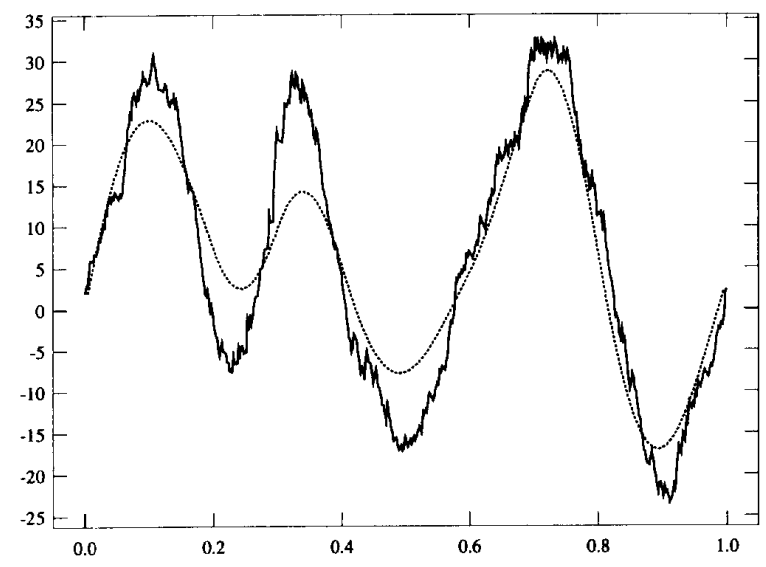

Fig. 10. Example 5: $z(t)$ (solid line) and $\mu(t)$ (dotted line).

and large values of $c$ can be explained heuristically by noting that since $D=E+c F, \alpha D \approx \alpha E$ for $c \approx 0$, and $\alpha D \approx \alpha c F$ for $c \approx \infty$.

In our simulations we have found that for $c$-values in the linear part of the $P_{i}(c)$-curve, good estimates of $\lambda$ are obtained by taking $\alpha=P_{i}(c)$. In fact, as $c$ varies over this linear region, the estimates

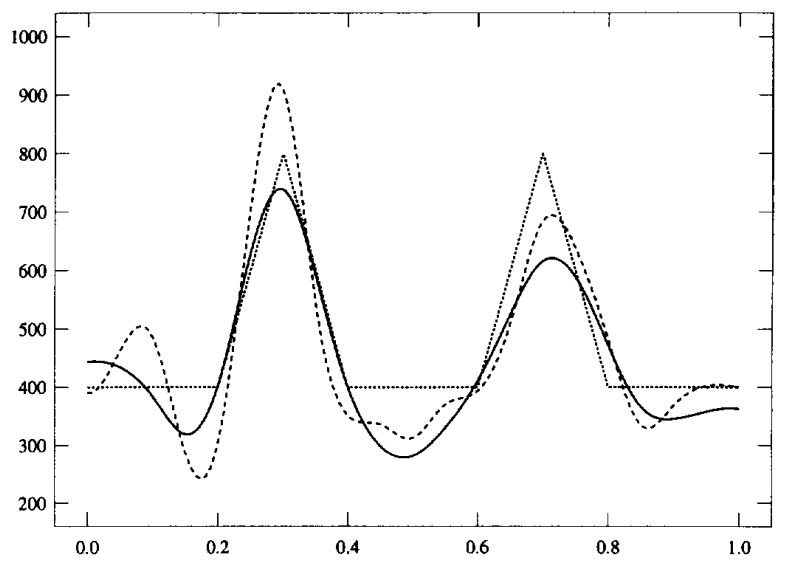

Fig. 11. Example $5: \bar{\lambda}$ corresponding to $\left(c_{1}, \alpha_{1}\right)$ (solid line), $\tilde{\lambda}$ corresponding to $\left(c_{3}, \alpha_{3}\right)$ (dashed line), and $\lambda$ (dotted line).

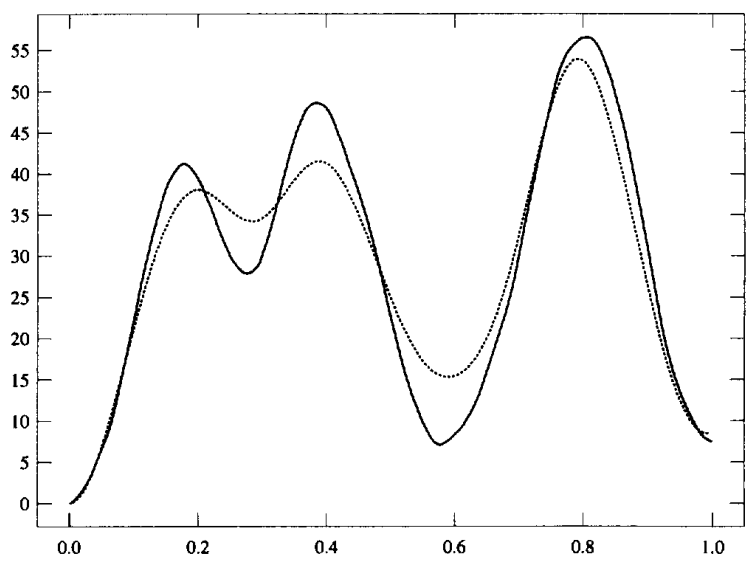

Fig. 12. Example 6: $z(t)$ (solid line) and $\mu(t)$ (dotted line).

corresponding to the pairs $/ c, \alpha)=\left(c, P_{i}(c) /\right.$ all look very similar; since we have found that the condition number of the matrix $D$ increases with $c$ in this region, $c$ should not be too large. Now, in some cases, associated with $\lambda$-functions that have a lot of "detail," we have found that the $P_{1}(c)$-curves look like the one shown in Fig. 6 . 


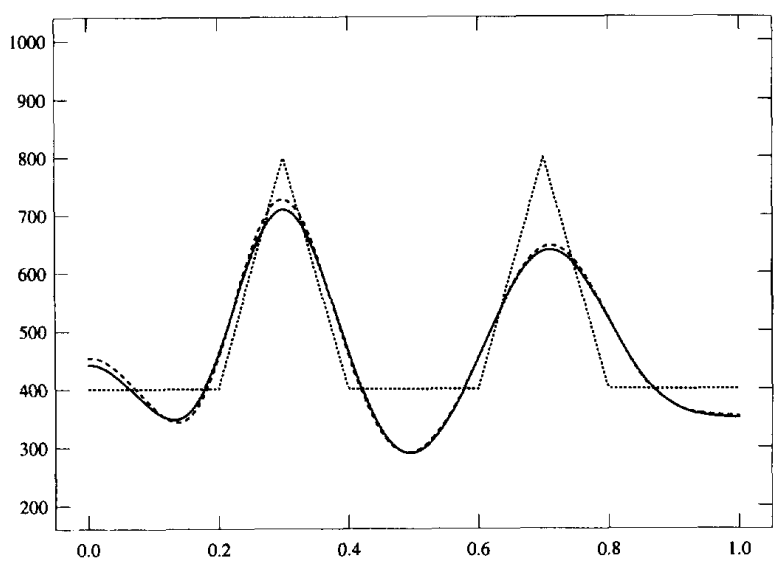

Fig. 13. Example $6: \bar{\lambda}$ corresponding to $\left(c_{1}, \alpha_{1}\right)$ (solid line), $\bar{\lambda}$ corresponding to $\left(c_{3}, \alpha_{3}\right)$ (dashed line), and $\lambda$ (dotted line).

In such cases, $P_{1}(c)$ is a satisfactory value of $\alpha$ over the region between the "flat" part and the "basin" of the curve.

Let $c_{i}$ denote a satisfactory value of $c$ chosen by examining the $P_{i}(c)$-curve. We then take $\alpha_{i}:=P_{i}\left(c_{i}\right), i=1,3$, in place of $\alpha$ in (2.3), and thus obtain the corresponding estimate of $\lambda$.

\section{NUMERICAL EXAMPLES}

We now present six numerical examples of the estimation of $\lambda$ for different $\lambda$ 's and different $h$ 's, and using (2.3) when the pair $(c, \alpha)$ is chosen as in Section VI. For each example, we generated $\Lambda:=\int_{0}^{1} \lambda(\tau) d \tau$ independent random points over $\mathcal{T}_{1}=[0,1]$ with density $\lambda(\tau) / \Lambda$, and then used $h$ to obtain $z$. We took $K=50$ uniformly-spaced samples of $z$ over $\mathcal{T}_{2}=[0,1]$ to form the vector $z$, and set $W=d I$, where $d=1 / K$. We took $\mathcal{B}$ as the span of the $M=21$ cubic B-splines [1] generated by the NAG [7] subroutine E02BCF when there are 4 coincident knots at both endpoints of $[0$ $1]$ and 17 internal knots at $0.1,0.15, \cdots, 0.85,0.9$.

In Examples 1-3, we took $h(t)=\exp (-3 t), t \geq 0$, and used the different $\lambda$ 's referred to in Table I (also listing $\Lambda$ ). This table refers to the figures showing $P_{i}(c)$ and $\tilde{\lambda}$, and lists the corresponding pairs $\left(c_{i}, \alpha_{i}\right)$. The importance of the parameter $c$ can be seen by looking at Fig. 3, which shows the estimate obtained under the setup of Example 1 when $c=0$ and $\alpha=P_{1}(0)=2.207 \times 10^{-3}$.

In Examples 4-6, we let $\lambda$ be shown as in Fig. $9(\Lambda=480)$, and used the different causal $h$-functions listed in Table II. This table refers to the figures showing $z, \mu$ [cf. (1.1)], and $\tilde{\lambda}$, and lists the corresponding pairs $\left(c_{i}, \alpha_{i}\right)$.

These examples cover low and high-intensity $\lambda$ 's, low and highvariation $\lambda$ 's, and a variety of impulse-response functions $h$. Based on these results, we see that both $\left(c_{1}, \alpha_{1}\right)$ and $\left(c_{3}, \alpha_{3}\right)$ allow one to recover detail in $\lambda$, but $\left(c_{1}, \alpha_{1}\right)$ does a better job of suppressing spurious oscillations.

\section{REFERENCES}

[1] C. de Boor, A Practical Guide to Splines. New York: Springer-Verlag, 1987

[2] P. Craven and G. Wahba, "Smoothing noisy data with spline functions," Numer. Math., vol. 31, pp. 377-403, 1979.
[3] C. W. Groetsch, The Theory of Tikhonov Regularization for Fredholm Equations of the First Kind. Boston, MA: Pitman, 1984

[4] T. J. Holmes, "Expectation-maximization restoration of band-limited, truncated point-process intensities with application in microscopy," $J$. Opt. Soc. Am. A, vol. 6, pp. 1006-1014, 1989.

[5] E. Masry, "Multivariate probability density deconvolution for stationary random processes," IEEE Trans. Inform. Theory, vol. IT-37, pp. 1105-1115, 1991.

[6] M. I. Miller and D. L. Snyder, "The role of likelihood and entropy in incomplete-data problems: Applications to estimating point-process intensities and Toeplitz constrained covariances," Proc. IEEE, vol. 75 , pp. 892-907, 1987.

[7] The NAG Fortran Library Manual, mark 15, vol. 3. The Numerical Algorithms Group Limited, 1991

[8] M. Z. Nashed, "Operator-theoretic and computational approaches to ill-posed problems with applications to antenna theory," IEEE Trans. Antennas Propagat., vol. AP-29, pp. 220-231, 1981.

[9] F. O'Sullivan, "A statistical perspective on ill-posed inverse problems," Statist. Sci., vol. 1, pp. 502-527, 1986.

[10] A. Papoulis, Probability, Random Variables, and Stochastic Processes. New York: McGraw-Hill, 1991

[11] L. Stefanski and R. J. Carroll, "Deconvoluting kernel density estimators," Statistics, vol. 21, pp. 169-184, 1990.

[12] G. Wahba, "Ill-posed problems: Numerical and statistical methods for mildly, moderately and severely ill-posed problems with noisy data," in Proc. Int. Conf. Ill-Posed Problems, Newark, NJ, Oct. 1979.

[13] _ "Spline models for observational data," in CBMS-NSF Regional Conference Series in Applied Mathematics, Philadelphia, SIAM, 1990, vol. 59 .

\section{Complex Hybrid Correlators: An Extended Analysis of their Performance}

Gaetano Giunta

Abstract-Discrete-time hybrid correlators were recently devised and their accuracy was provided in a closed form for the simplest cases of instantaneous nonlinearities, in the reference case of complex Gaussian stationary processes. This correspondence extends the performance analysis to finer phase nonlinearities.

\section{INTRODUCTION}

Correlation is one basic function in signal analysis, required in several detection and estimation problems, such as spectral analysis or time delay estimation. Some fast hybrid methods to estimate correlation working on complex processes are based on magnitude hard limiting and phase coarse quantization of one input signal [1] Besides the computational advantages of such multiplication-free algorithms, especially in hardware-based or on-chip implementation, these methods are useful when the magnitude of one signal is subjected to system gain fluctuations or dynamic range problems which cannot be accornmodated by automatic gain control devices.

Only some of the discrete-phase hybrid correlators (namely: 2,4 , and $\infty$-phases) have been so far analyzed in the literature [2], [3]. This correspondence aims to fill such gap by providing the mean square error (MSE) for any number of bits of the phase quantizer. In fact, the MSE results a function of the fourfold hybrid moments,

Manuscript received November 11, 1993; revised December 2, 1994.

The author is with the INFO-COM Department, University of Rome La Sapienza, Via Eudossiana 18, 00184 Rome, Italy.

IEEE Log Number 9411185. 